\title{
Third order Lovelock black branes in the presence of a nonlinear electromagnetic field
}

\author{
S. H. Hendi ${ }^{1,2 a}$, S. Panahiyan ${ }^{1}$, and H. Mohammadpour ${ }^{1}$ \\ 1 Physics Department and Biruni Observatory, Shiraz University, Shiraz 71454, Iran \\ 2 Research Institute for Astrophysics and Astronomy of Maragha (RIAAM), P.O. Box 55134-441, Maragha, Iran
}

Received: date / Revised version: date

\begin{abstract}
We consider third order Lovelock gravity coupled to an U(1) gauge field for which its Lagrangian is given by a power of Maxwell invariant. In this paper, we present a class of horizon flat rotating black branes and investigate their geometrical properties and the effect of nonlinearity on the solutions. We use some known formulas and methods to calculate thermodynamic and conserved quantities. Finally, we check the satisfaction of the first law of thermodynamics.
\end{abstract}

PACS. PACS-key discribing text of that key - PACS-key discribing text of that key

\section{Introduction}

Lovelock gravity is one of the higher derivative gravity theories, natural generalization of Einstein's General Relativity, introduced by David Lovelock [1,2,3] in 1971

$$
I_{G}=\int d^{d} x \sqrt{-g} \sum_{k=0}^{d / 2} \alpha_{k} £_{k},
$$

where $\left(\alpha_{k}\right)$ is an arbitrary constant and $\left(£_{k}\right)$ is the Euler density of a $2 k$-dimensional manifold

$$
£_{k}=\delta_{\rho_{1} \sigma_{1} \ldots \rho_{k} \sigma_{k}}^{\mu_{1} \nu_{1} \ldots \mu_{k} \nu_{k}} R_{\mu_{1} \nu_{1}}^{\rho_{1} \sigma_{1}} \ldots R_{\mu_{k} \nu_{k}}^{\rho_{k} \sigma_{k}}
$$

In this equation, $\delta_{\rho_{1} \sigma_{1} \ldots \rho_{k} \sigma_{k}}^{\mu_{1} \nu_{1} \ldots \mu_{k} \nu_{k}}$ is the generalized totally antisymmetric Kronecker delta and $\left(R_{\mu \nu}^{\rho \sigma}\right)$ is the Riemann tensor.

It has been shown that gravitational field equation, arising from variation of the action 1, only contains the terms with at most second order derivatives of metric which indicates the quantization of linearized Lovelock theory is free of ghosts [4,5. Also, such theory may be used in the context of AdS/CFT correspondence to investigate the effects of including higher-curvature terms [6, 7, 8,9]. Another interesting property of this theory comes from the fact that there is a delicate relationship between Lovelock gravity and low energy effective action of heterotic string theory in addition to the Calabi-Yau compactifications of M-theory [4, 5].

On the other hand, it has been shown that loop corrections of gravity 10,11, 12, 13, 14 may be lead to a nonlinear Born-Infeld type Lagrangian [15,16] . Hoffmann was

\footnotetext{
a hendi@shirazu.ac.ir
}

the first one to attempt to couple the nonlinear electrodynamics with gravity 17. One of the current interesting subjects in black hole physics is the investigation of the effects of nonlinear electrodynamic fields on the gravitational field [18, 19, 20, 21, 22, 23, 24, 25, 26, 27, 28, 29, 30, 31, 32 33, 34, 35, 36, 37. Considering nonlinear model of electrodynamic fields provide a powerful laboratory to analyze the properties of the black hole solutions.

In this paper, we obtain $d$-dimensional rotating black brane solutions of the first four terms of Lovelock theory which are cosmological constant, Einstein, Gauss-Bonnet and third order Lovelock terms, in the presence of nonlinear electromagnetic field and analyze their properties. We should note that third order Lovelock term is an Euler density in six dimensions and in order to have the contribution of all the above terms in the field equation, the dimension of the spacetime should be equal to or larger than seven. These solutions can be regarded as a generalization of the Lovelock-Maxwell gravity [38,39]. We discuss some geometrical and thermodynamic properties, like curvature, temperature and entropy. We also make some comments on the effects of nonlinearity parameter and Lovelock coefficients.

\section{Field Equations}

The action of third order Lovelock gravity in the presence of power-Maxwell-invariant source may be written as

$$
\begin{aligned}
\mathcal{I}= & -\frac{1}{16 \pi} \int_{\mathcal{M}} d^{d} x \sqrt{-g}\left[R-2 \Lambda+\alpha_{2} \mathcal{L}_{2}+\alpha_{3} \mathcal{L}_{3}+\right. \\
& \left.(\kappa \mathcal{F})^{s}\right]+\mathcal{I}_{b},
\end{aligned}
$$


where $R$ is the Ricci scalar, $\Lambda$ is the cosmological constant, $\alpha_{i}$ 's are Lovelock coefficients, $\mathcal{L}_{2}$ and $\mathcal{L}_{3}$ denote the GaussBonnet Lagrangian and the third order Lovelock term, given as

$$
\begin{gathered}
\mathcal{L}_{2}=R_{\mu \nu \gamma \delta} R^{\mu \nu \gamma \delta}-4 R_{\mu \nu} R^{\mu \nu}+R^{2} \\
\mathcal{L}_{3}=2 R^{\mu \nu \sigma \kappa} R_{\sigma \kappa \rho \tau} R^{\rho \tau}{ }_{\mu \nu}+8 R^{\mu \nu}{ }_{\sigma \rho} R_{\nu \tau}^{\sigma \kappa} R_{\mu \kappa}^{\rho \tau}+ \\
24 R^{\mu \nu \sigma \kappa} R_{\sigma \kappa \nu \rho} R_{\mu}^{\rho}+3 R R^{\mu \nu \sigma \kappa} R_{\sigma \kappa \mu \nu}-12 R R_{\mu \nu} R^{\mu \nu} \\
+24 R^{\mu \nu \sigma \kappa} R_{\sigma \mu} R_{\kappa \nu}+16 R^{\mu \nu} R_{\nu \sigma} R_{\mu}^{\sigma+R^{3}} .
\end{gathered}
$$

The last term in the first integral is the Lagrangian of power-Maxwell-invariant theory, where $\kappa$ is an arbitrary constant and $\mathcal{F}$ is the Maxwell invariant which is equal to $F_{\mu \nu} F^{\mu \nu}\left(F_{\mu \nu}=\partial_{\mu} A_{\nu}-\partial_{\nu} A_{\mu}\right.$ is the electromagnetic tensor field and $A_{\mu}$ is the vector potential) and the last term is the boundary term which is chosen such that the variational principle is well defined. Varying the action (3) with respect to $g_{\mu \nu}$ and $A_{\mu}$, one can obtain the gravitational as well as the electromagnetic field equations

$$
\begin{gathered}
G_{\mu \nu}^{(1)}+\Lambda g_{\mu \nu}+\alpha_{2} G_{\mu \nu}^{(2)}+\alpha_{3} G_{\mu \nu}^{(3)}= \\
-2\left[\kappa s F_{\mu \rho} F_{\nu}^{\rho}(\kappa \mathcal{F})^{s-1}-\frac{1}{4} g_{\mu \nu}(\kappa \mathcal{F})^{s}\right], \\
\partial_{\mu}\left(\sqrt{-g}(\kappa \mathcal{F})^{s-1} F^{\mu \nu}\right)=0
\end{gathered}
$$

where $G_{\mu \nu}^{(1)}=R_{\mu \nu}-\frac{R}{2} g_{\mu \nu}$ is just the Einstein tensor, and $G_{\mu \nu}^{(2)}$ and $G_{\mu \nu}^{(3)}$ are the second and third order Lovelock tensors obtained as

$$
\begin{aligned}
G_{\mu \nu}^{(2)}= & 2\left(R_{\mu \sigma \kappa \tau} R_{\nu}{ }^{\sigma \kappa \tau}-2 R_{\mu \rho \nu \sigma} R^{\rho \sigma}-2 R_{\mu \sigma} R_{\nu}^{\sigma}+R R_{\mu \nu}\right) \\
& -\frac{1}{2} \mathcal{L}_{2} g_{\mu \nu},
\end{aligned}
$$

$$
\begin{aligned}
G_{\mu \nu}^{(3)}= & -3\left(4 R^{\tau \rho \sigma \kappa} R_{\sigma \kappa \lambda \rho} R_{\nu \tau \mu}^{\lambda}-8 R_{\lambda \sigma}^{\tau \rho} R_{\tau \mu}^{\sigma \kappa} R_{\nu \rho \kappa}^{\lambda}+\right. \\
& 2 R_{\nu}{ }^{\tau \sigma \kappa} R_{\sigma \kappa \lambda \rho} R^{\lambda \rho}{ }_{\tau \mu}-R^{\tau \rho \sigma \kappa} R_{\sigma \kappa \tau \rho} R_{\nu \mu}+ \\
& 8 R^{\tau}{ }_{\nu \sigma \rho} R^{\sigma \kappa}{ }_{\tau \mu} R^{\rho}{ }_{\kappa}+8 R_{\nu \tau \kappa}^{\sigma} R^{\tau \rho}{ }_{\sigma \mu} R^{\kappa}{ }_{\rho} \\
& +4 R_{\nu}{ }^{\tau \sigma \kappa} R_{\sigma \kappa \mu \rho} R_{\tau}^{\rho}-4 R_{\nu}{ }^{\tau \sigma \kappa} R_{\sigma \kappa \tau \rho} R^{\rho}{ }_{\mu}+ \\
& 4 R^{\tau \rho \sigma \kappa} R_{\sigma \kappa \tau \mu} R_{\nu \rho}+2 R R_{\nu}{ }^{\kappa \tau \rho} R_{\tau \rho \kappa \mu}+ \\
& 8 R^{\tau}{ }_{\nu \mu \rho} R^{\rho}{ }_{\sigma} R^{\sigma}{ }_{\tau}+8 R^{\tau}{ }_{\nu \mu \rho} R^{\rho}{ }_{\sigma} R^{\sigma}{ }_{\tau}- \\
& 8 R^{\sigma}{ }_{\nu \tau \rho} R^{\tau}{ }_{\sigma} R_{\mu}^{\rho}-8 R^{\tau \rho}{ }_{\sigma \mu} R^{\sigma}{ }_{\tau} R_{\nu \rho}-4 R R_{\nu \mu \rho}^{\tau} R_{\tau}^{\rho} \\
& +4 R^{\tau \rho} R_{\rho \tau} R_{\nu \mu}-8 R_{\nu}^{\tau} R_{\tau \rho} R^{\rho}{ }_{\mu}+4 R R_{\nu \rho} R_{\mu}^{\rho}{ }_{\mu} \\
& \left.-R^{2} R_{\nu \mu}\right)-\frac{1}{2} \mathcal{L}_{3} g_{\mu \nu} .
\end{aligned}
$$

The Lovelock supplementation, $\mathcal{I}_{b}$, is Gibbons-HawkingYork boundary term which may be written as

$$
\mathcal{I}_{b}=-\frac{1}{8 \pi} \int_{\partial \mathcal{M}} d^{d-1} x \sqrt{-\gamma}\left[K+\alpha_{2} L_{2 b}+\alpha_{3} L_{3 b}\right],
$$

where

$$
L_{2 b}=2\left(J-2 \widehat{G}_{a b}^{(1)} K^{a b}\right)
$$

$$
\begin{aligned}
L_{3 b}= & 3\left(P-2 \widehat{G}_{a b}^{(2)} K^{a b}-12 \widehat{R}_{a b} J^{a b}+2 \widehat{R} J-\right. \\
& \left.4 K \widehat{R}_{a b c d} K^{a c} K^{b d}-8 \widehat{R}_{a b c d} K^{a c} K_{e}^{b} K^{e d}\right) .
\end{aligned}
$$

In these equations $\gamma_{\mu \nu}$ and $K$ are, respectively, induced metric and the trace of extrinsic curvature of boundary, $\widehat{G}_{a b}^{(1)}$ and $\widehat{G}_{a b}^{(2)}$ denote the $(d-1)$-dimensional Einstein and second order Lovelock tensors (Eq. (8)) of the metric $\gamma_{a b}$ while $J$ and $P$ are the traces of

$$
\begin{aligned}
J_{a b}= & \frac{1}{3}\left(2 K K_{a c} K_{b}^{c}+K_{c d} K^{c d} K_{a b}-\right. \\
& \left.2 K_{a c} K^{c d} K_{d b}-K^{2} K_{a b}\right),
\end{aligned}
$$

and

$$
\begin{aligned}
P_{a b}= & \frac{1}{5}\left\{\left[K^{4}-6 K^{2} K^{c d} K_{c d}+8 K K_{c d} K_{e}^{d} K^{e c}-\right.\right. \\
& \left.6 K_{c d} K^{d e} K_{e f} K^{f c}+3\left(K_{c d} K^{c d}\right)^{2}\right] K_{a b}- \\
& \left(4 K^{3}-12 K K_{e d} K^{e d}+8 K_{d e} K_{f}^{e} K^{f d}\right) K_{a c} K_{b}^{c}- \\
& 24 K K_{a c} K^{c d} K_{d e} K_{b}^{e}+12\left(K^{2}-K_{e f} K^{e f}\right) K_{a c} K^{c d} K_{d b} \\
& \left.+24 K_{a c} K^{c d} K_{d e} K^{e f} K_{b f}\right\}
\end{aligned}
$$

\section{The $d$-dimensional Charged Rotating Black Branes}

Now, we should consider a rotating spacetime and investigate its properties. Since the rotation group and the number of independent rotation parameters in $d$-dimensions are, respectively, $S O(d-1)$ and the integer part of $(d-$ $1) / 2$, one of the rotating metrics with $k \leq[(d-1) / 2]$ rotation parameters and zero curvature boundary may be written as

$$
\begin{aligned}
d s^{2}= & -f(r)\left(\Xi d t-\sum_{i=1}^{k} a_{i} d \phi_{i}\right)^{2}+\frac{d r^{2}}{f(r)}+ \\
& \frac{r^{2}}{l^{4}} \sum_{i=1}^{k}\left(a_{i} d t-\Xi l^{2} d \phi_{i}\right)^{2}-\frac{r^{2}}{l^{2}} \sum_{i<j}^{k}\left(a_{i} d \phi_{j}-a_{j} d \phi_{i}\right)^{2} \\
& +r^{2} \sum_{i=1}^{d-2-k} d x_{i}^{2}
\end{aligned}
$$

where $\Xi=\sqrt{1+\sum_{i}^{k} a_{i}^{2} / l^{2}}$ and the angular coordinates are in the range $0 \leq \phi_{i} \leq 2 \pi$. Using the suitable gauge potential ansatz

$$
\left.A_{\mu}=h(r)\left(\Xi \delta_{\mu}^{0}-a_{i} \delta_{\mu}^{i}\right) \text { (no sum on } i\right),
$$

in Eq. (7) leads to the following differential equations

$$
E q_{h}=(2 s-1) r h^{\prime \prime}+(d-2) h^{\prime}=0,
$$

with the following solutions

$$
h(r)=\left\{\begin{array}{cc}
-q \ln r & \text { for } s=\frac{d-1}{2} \\
-q r^{(2 s-d+1) /(2 s-1)} & \text { otherwise }
\end{array},\right.
$$


where prime and double prime denote first and second derivatives with respect to $r$, respectively and $q$ is an integration constant. It is worthwhile to note that, one can choose $s=d / 4$ to obtain inverse-square electric field. In this case the expression of the electric field does not depend on the dimension and its value coincides with the Reissner-Nordström solution in four dimensions. In addition, in arbitrary dimensions with $s=(d-1) / 2$, the gauge potential is logarithmic and it coincides with the charged BTZ solution. In order to have a sensible asymptotic structure, the electromagnetic field should vanish for large values of $r$. This condition leads to $s>1 / 2$. In this paper we would like to investigate the solutions of general case (i.e. $s>1 / 2$ and $s \neq(d-1) / 2)$. In addition, one can find that $\mathcal{F}=-2\left(\frac{d h(r)}{d r}\right)^{2}$ and the power of Maxwell invariant, $(\kappa \mathcal{F})^{s}$, may be imaginary for positive $\kappa$, when $s$ is fractional. Therefore, we set $\kappa=-1$ to have real solutions without loss of generality.

To find the metric function $f(r)$, we should consider the components of Eq. (6). At first, we consider the static case $\left(a_{i}=0\right)$. One may show that the $t t$-component of Eq. (6) may be written as

$$
\begin{aligned}
E q_{t t}= & \frac{r}{4}\left[6 ! \alpha_{3}\left(\begin{array}{c}
d-2 \\
5
\end{array}\right) f^{2}-4 ! \alpha_{2}\left(\begin{array}{c}
d-2 \\
3
\end{array}\right) r^{2} f+2(d-2) r^{4}\right] f^{\prime} \\
& +\left[6 !\left(\begin{array}{c}
d-2 \\
6
\end{array}\right) \alpha_{3} f^{2}-4 ! \alpha_{2}\left(\begin{array}{c}
d-2 \\
4
\end{array}\right) r^{2} f+2\left(\begin{array}{c}
d-2 \\
2
\end{array}\right) r^{4}\right] \frac{f}{2} \\
& +\Lambda r^{6}+\frac{(2 s-1)\left(2 h^{\prime 2}\right)^{s} r^{6}}{2}=0
\end{aligned}
$$

where $\left(\begin{array}{c}p \\ q\end{array}\right)=\frac{p !}{(p-q) ! q !}$. Other nonzero components of Eq. (6) are a combination of $\frac{d E q_{t t}}{d r}, E q_{t t}$ and $E q_{h}$. For example $x_{i} x_{i}$-component of Eq. (6) can be written as

$$
E q_{x_{i} x_{i}}=\frac{d}{d r} E q_{t t}+\frac{(d-8)}{r} E q_{t t}-\frac{s r^{5}\left(2 h^{\prime 2}\right)^{s}}{h^{\prime}} E q_{h}=0
$$

Since $E q_{h}$ vanishes for obtained $h(r)$, it is sufficient to solve $E q_{t t}=0$. For simplicity, we choose a special case

$$
\alpha_{3}=\frac{(d-3)(d-4) \alpha_{2}^{2}}{3(d-5)(d-6)} .
$$

The solution of Eq. (19) can be written as

$$
f(r)=\frac{r^{2}}{(d-3)(d-4) \alpha_{2}}\left[1-g(r)^{1 / 3}\right],
$$

where

$$
\begin{aligned}
g(r)= & +\frac{6(d-3)(d-4) \alpha_{2} \Lambda}{(d-1)(d-2)}+\frac{3(d-3)(d-4) \alpha_{2} M}{r^{d-1}} \\
& -\frac{3(d-3)(d-4) \alpha_{2}(2 s-1)^{2} L(r)}{(d-2)(2 s-d+1)}, \\
L(r)= & \left(\frac{2 q^{2}(2 s-d+1)^{2}}{(2 s-1)^{2} r^{2(d-2) /(2 s-1)}}\right)^{s},
\end{aligned}
$$

and $M$ is an integration constant. We should note that, in addition to static components of Eq. (6), Eq. (21) satisfies all nonstatic components of Eq. (6). In addition, it is notable that this solution reduces to the solution of Ref. [39] for $s=1$, as it should be.

Due to the fact that the power of $r$ in the denominator of $L(r)$ is bigger than unity for arbitrary $d>6$ and $s>$ $1 / 2, L(r)$ vanishes for large values of $r$. Therefore in order to investigate the asymptotic behavior of the solutions, one can consider the vacuum solutions $(M=q=0)$

$$
f(r)=\frac{r^{2}\left(1-\left[1+\frac{6(d-3)(d-4) \alpha_{2} \Lambda}{(d-1)(d-2)}\right]^{\frac{1}{3}}\right)}{(d-3)(d-4) \alpha_{2}} .
$$

Considering the former equation, it is easy to find that the asymptotic behavior of the solutions is AdS for negative $\Lambda$. One can put $\Lambda=-(d-1)(d-2) / 2 l^{2}$ in Eq. (23) to find the effective cosmological constant of the solutions

$$
\Lambda_{\mathrm{eff}}=-\frac{(d-1)(d-2)\left(1-\left[1-\frac{3(d-3)(d-4) \alpha_{2}}{l^{2}}\right]^{\frac{1}{3}}\right)}{2(d-3)(d-4) \alpha_{2}} .
$$

Now, we look for the essential singularity(ies). It is easy to show that the Kretschmann scalar of the metric (15) is

$$
R_{\alpha \beta \gamma \delta} R^{\alpha \beta \gamma \delta}=f^{\prime \prime 2}+2(d-2) \frac{f^{\prime 2}}{r^{2}}+2(d-2)(d-3) \frac{f^{2}}{r^{4}} .
$$

After some algebraic manipulation, one can show that the Kretschmann scalar (25) with metric function (21) diverges at $r=0$ and therefore there is an essential timelike singularity located at $r=0$. In order to investigate the existence of horizon(s), one should find the root(s) of $g^{r r}=f(r)=0$. In addition, we can use the fact that the temperature of the extreme black brane is zero. In the case of extreme black brane solution, the only real root of $f(r)$ is its minimum. So, it is easy to find that the mass parameter for this case becomes

$$
m_{\mathrm{ext}}=\frac{4 \Lambda s\left(\frac{-2 \Lambda}{\left(\frac{2(2 s-d+1)^{2} q_{\mathrm{ext}}^{2}}{(2 s-1)^{2}}\right)^{s}(2 s-1)}\right)^{\frac{(d-1)(2 s-1)}{[2 s(d-2)]}}}{(2 s-d+1)(d-1)} .
$$

Finally, we should note that obtained solutions may be interpreted as black branes with inner and outer event horizons provided $m>m_{\text {ext }}$, extreme black brane for $m=m_{\text {ext }}$ and naked singularity otherwise (see Fig. 1 for more details). In addition, it is desirable to investigate the effects of the Lovelock parameter, $\alpha_{2}$ and the nonlinearity parameter, $s$. For example, figures 2 and 3 show that both $\alpha_{2}$ and $s$ affect on the minimum value of $f(r)$. Also, these figures confirm that in spite of $\alpha_{2}$, the nonlinearity parameter, $s$ has a considerable role on the values of inner and outer horizons.

\section{Thermodynamic and Conserved Quantities and the first law}

Now, we are in a position to calculate the thermodynamic and conserved quantities of the solutions. The Hawking 


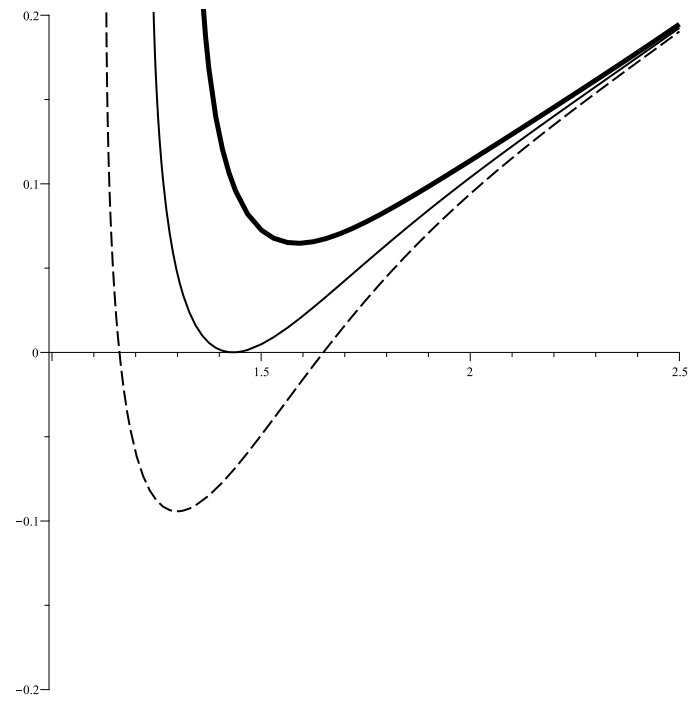

Fig. 1. $f(r)$ versus $r$ for $d=10, q=1, \Lambda=-1, \alpha_{2}=0.1$, $s=2$, and $M=3.52<M_{\text {ext }}$ (bold line), $M=4.52=M_{\text {ext }}$ (solid line) and $M=5.52>M_{\text {ext }}$ (dashed line).

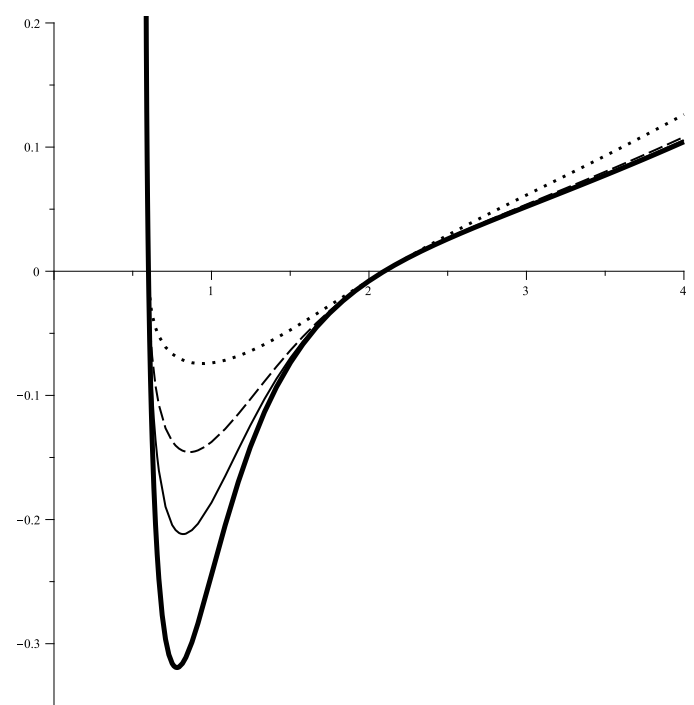

Fig. 2. $f(r)$ versus $r$ for $d=7, q=1, \Lambda=-0.1, M=1$, $s=2$, and $\alpha_{2}=0.05$ (bold line), $\alpha_{2}=0.2$ (solid line), $\alpha_{2}=0.5$ (dashed line) and $\alpha_{2}=2$ (dotted line).

temperature and angular velocities of the event horizon of the black branes may be calculated by requiring the absence of conical singularity at the horizon in the Euclidean sector of the solutions. Considering the analytic continuation of the metric by setting $t \longrightarrow i \tau$ and $a_{i} \longrightarrow i a_{i}$, and regularity at event horizon $\left(r=r_{+}\right)$help us to obtain the Hawking temperature and the angular velocities of the black branes

$$
\begin{aligned}
T_{+}= & \beta_{+}^{-1}=\frac{f^{\prime}\left(r_{+}\right)}{4 \pi \Xi}= \\
& \frac{r_{+}\left[(1-2 s)\left(\frac{2 q^{2}(2 s-d+1)^{2}}{(2 s-1)^{2} r_{+}^{2(d-2) /(2 s-1)}}\right)^{s}-2 \Lambda\right]}{4(d-2) \pi \Xi}
\end{aligned}
$$

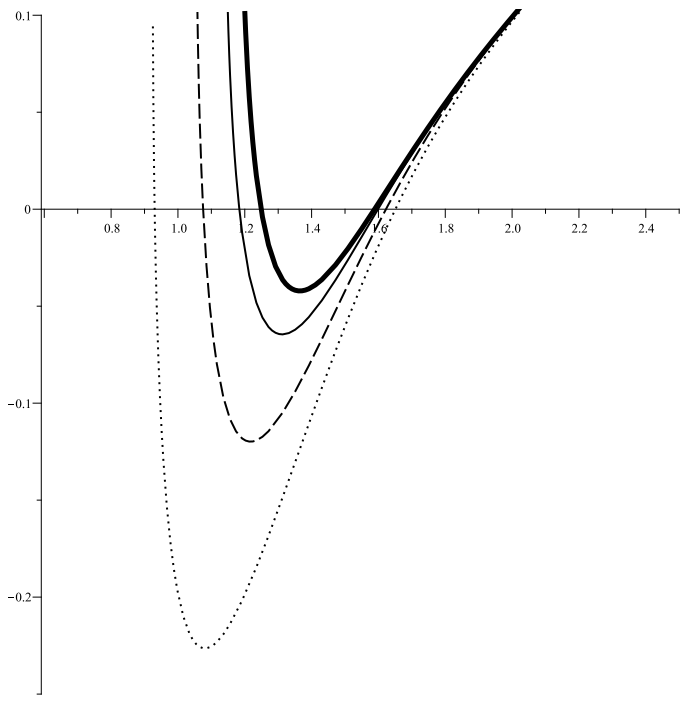

Fig. 3. $f(r)$ versus $r$ for $d=10, q=1, \Lambda=-1, M=5$, $\alpha_{2}=0.1$, and $s=2$ (bold line), $s=2.1$ (solid line), $s=2.2$ (dashed line) and $s=2.3$ (dotted line).

$$
\Omega_{i}=\frac{a_{i}}{\Xi l^{2}}
$$

Using the fact that the temperature of the extreme black branes vanishes, one can write the horizon radius of the extreme black brane

$$
r_{\mathrm{ext}}^{2 s(d-2) /(2 s-1)}=\frac{\left(\frac{1}{2}-s\right)}{\Lambda}\left(\frac{2 q_{\mathrm{ext}}^{2}(2 s-d+1)^{2}}{(2 s-1)^{2}}\right)^{s} .
$$

In order to obtain the electric charge of the solution, we use the generalized Maxwell equation (7). Calculating the flux of the electric field at infinity leads to the electric charge per unit volume $V_{d-2}$

$$
Q=\frac{2^{s} \Xi s}{8 \pi}\left(\frac{(2 s-d+1) q}{2 s-1}\right)^{2 s-1} .
$$

The electric potential $U$, measured at infinity with respect to the horizon, is calculated by the following relation

$$
U=\left.A_{\mu} \chi^{\mu}\right|_{r \rightarrow \infty}-\left.A_{\mu} \chi^{\mu}\right|_{r=r_{+}},
$$

where $\chi=\partial_{t}+\sum_{i}^{k} \Omega_{i} \partial_{\phi_{i}}$ is the Killing vector. The electric potential will be

$$
U=\frac{q}{\Xi} r_{+}^{(2 s-d+1) /(2 s-1)} .
$$

Now, we should calculate the total mass density of the black branes. We may obtain finite mass through the use of the counterterm method [40. One can show that for obtained solutions with flat boundary, $\widehat{R}_{a b c d}(\gamma)=0$, the finite action is 39,41

$$
\mathcal{I}_{\text {finite }}=\mathcal{I}+\frac{1}{8 \pi} \int_{\partial \mathcal{M}} d^{d-1} x \sqrt{-\gamma}\left(\frac{d-2}{l_{\text {eff }}}\right) .
$$


It is notable that that this counterterm has exactly the same form as the counterterm in Einstein gravity for a spacetime with zero curvature boundary in which $l$ is replaced by $l_{e f f}$. After some algebraic manipulation, one can show that $l_{\text {eff }}$ is given by

$$
\begin{aligned}
l_{e f f} & =\frac{15 \sqrt{(1-\lambda)(d-3)(d-4) \alpha_{2}}}{9\left(1+\frac{(d-3)(d-4) \alpha_{2}}{l^{2}}\right)-(2+\lambda)^{2}}, \\
\lambda & =\left(1-\frac{3(d-3)(d-4) \alpha_{2}}{l^{2}}\right)^{1 / 3},
\end{aligned}
$$

which reduces to $l$ as $\alpha$ goes to zero. Using Eqs. (3) and (33) with Eq. (34), the finite action per unit volume $V_{d-2}$ can be obtained as

$$
I=\frac{\beta_{+} r_{+}^{d-1}\left[\frac{\Lambda}{(d-1)}+\frac{2^{s-1} q^{2 s}(2 s-2 d+3)}{r_{+}^{2 s(d-2) /(2 s-1)}\left(\frac{(2 s-d+1)}{2 s-1}\right)^{1-2 s}}\right]}{8 \pi(d-2)} .
$$

Having the total finite action, we can use the Brown-York method of quasilocal definition [42] to introduce the following divergence-free stress-energy tensor

$$
\begin{aligned}
T^{a b}= & \frac{1}{8 \pi}\left\{\left(K^{a b}-K \gamma^{a b}\right)+2 \alpha_{2}\left(3 J^{a b}-J \gamma^{a b}\right)\right. \\
& \left.+3 \alpha_{3}\left(5 P^{a b}-P \gamma^{a b}\right)+\frac{d-2}{l_{e f f}} \gamma^{a b}\right\} .
\end{aligned}
$$

It is easy to find that $\partial / \partial t$ and $\partial / \partial \phi^{i}$ are the Killing vectors of the metric (15) and therefore the associated conserved quantities are the mass and angular momentum, which are

$$
\begin{aligned}
M & =\frac{V_{d-2}}{16 \pi} m\left[(d-1) \Xi^{2}-1\right], \\
J_{i} & =\frac{V_{d-2}(d-1)}{16 \pi} \Xi m a_{i} .
\end{aligned}
$$

We should note that for static solutions $\left(a_{i}=0\right.$ and therefore $\Xi=1$ ), the angular momentum vanishes, and thus the $a_{i}$ 's are the rotational parameters of the spacetime.

In order to complete this section, we should calculate the entropy of the black branes. Since the area law is applicable to black holes (branes) in Einstein gravity [43, 44, 45, 46, 47, 48, 49, we calculate the entropy through the use of Gibbs-Duhem relation

$$
\mathcal{S}=\frac{1}{T}\left(M-Q U-\sum_{i=1}^{k} \Omega_{i} J_{i}\right)-I .
$$

It is strightforward to calculate the entropy per unit volume $V_{d-2}$ by use of Eq. (39) with obtained thermodynamic and conserved quantities

$$
\mathcal{S}=\frac{\Xi}{4} r_{+}^{d-2}
$$

which confirms the entropy obeys the area law for our case (flat horizon).
Now, we are in a position to check the first law of thermodynamics. It has been found that the first law may be derived from Smarr(-type) formula for the total energy of black holes [50. In order to obtain Smarr-type formula, one can calculate the mass density as a function of the extensive quantities $\mathcal{S}, \mathbf{J}$, and $Q$. Using Eqs. (30), (37), (38), (40) and the fact that $r_{+}$is the largest root of $f(r)$, we obtain

$$
M(\mathcal{S}, \mathbf{J}, Q)=\frac{[(d-1) Z-1] J}{(d-1) l \sqrt{Z(Z-1)}},
$$

where $J=|\mathbf{J}|=\sqrt{\sum_{i}^{k} J_{i}^{2}}$ and $Z=\Xi^{2}$ is the positive real root of the following equation

$$
\begin{aligned}
& \frac{\left[\frac{2^{s-1}(d-1)(2 s-1)^{2}}{(2 s-d+1)}\left(\frac{2^{1-s}}{s} \frac{\pi Q}{\mathcal{S}}\right)^{\frac{2 s}{2 s-1}}+\Lambda\right] l(4 \mathcal{S})^{\frac{d-1}{d-2}}}{8 \pi(d-2) J}- \\
& \sqrt{\frac{Z^{\frac{1}{d-2}}}{Z-1}}=0 .
\end{aligned}
$$

Now, we can regard the mass $M(\mathcal{S}, \mathbf{J}, Q)$ as a function of extensive parameters and obtain the intensive parameters conjugate to them in the following manner

$$
T=\left(\frac{\partial M}{\partial \mathcal{S}}\right)_{J, Q}, \quad \Omega_{i}=\left(\frac{\partial M}{\partial J_{i}}\right)_{\mathcal{S}, Q}, \quad U=\left(\frac{\partial M}{\partial Q}\right)_{\mathcal{S}, J} .
$$

Using the chain rule, it is straightforward to show that the intensive quantities calculated by Eq. (43) coincide with Eqs. (27), (28) and (32), and hence we can deduce that our black brane solutions satisfy the first law of thermodynamics

$$
d M=T d \mathcal{S}+\sum_{i=1}^{k} \Omega_{i} d J_{i}+U d Q
$$

\section{CLOSING REMARKS}

In this paper, we regarded the first four terms of Lovelock gravity with a source of nonlinear electromagnetic field. We found a class of rotating solutions which may be interpreted as black branes with inner and outer horizons, extreme black branes or naked singularity.

We used the analytic continuation of the metric to calculate the Hawking temperature and angular velocities of the black branes. We then considered the Gauss's law to obtain the finite electric charge of the black branes. In addition, we calculated the finite action, mass and angular momentum by using the counterterm method. Since the area law can be applied to black holes (branes) in Einstein gravity, we calculated the entropy through the use of Gibbs-Duhem relation and found that the entropy obeys the area law.

The Smarr-type formula for the mass was also obtained as a function of extensive quantities to compute temperature, angular velocities and electric potential. It was then 
confirmed that the thermodynamic and conserved quantities satisfy the first law of thermodynamics.

Finally, it is also desirable to study the causal structure, the ratio of shear viscosity to entropy density, thermodynamic stability and dynamical properties of the black brane solutions derived here. Generalization of these solutions to various (nontrivial) horizon topologies, remain to be carried out in the future. In addition, It would be also interesting to investigate the effect of curvature-cubed Lagrangian [51,52] and quartic quasitopological gravity [53] with a nonlinear source. We hope to address these issues in the future.

Acknowledgement: We thank the referee for constructive comments. This work has been supported financially by Research Institute for Astronomy \& Astrophysics of Maragha (RIAAM).

\section{References}

1. D. Lovelock, J. Math. Phys. 12, 498 (1971)

2. D. Lovelock, J. Math. Phys. 13, 874 (1972)

3. A. Navarro and J. Navarro, J. Geom. Phys. 61, 1950 (2011)

4. B. Zwiebach, Phys. Lett. B 156, 315 (1985)

5. D. Boulware and S. Deser, Phys. Rev. Lett. 55, 2656 (1985)

6. M. Fukuma, S. Matsuura and T. Sakai, Prog. Theor. Phys. 105, $1017(2001)$

7. H. Saida and J. Soda, arXiv:gr-qc/0011095

8. X. O. Camanho and J. D. Edelstein, arXiv:0912.1944

9. T. Takahashi and J. Soda, [arXiv:1108.5041]

10. E. S. Fradkin and A. A. Tseytlin, Phys. Lett. B 163, 123 (1985)

11. E. Bergshoeff, E. Sezgin, C. N. Pope and P. K. Townsend, Phys. Lett. B 188, 70 (1987)

12. R. R. Metsaev, M. A. Rahmanov and A. A. Tseytlin, Phys. Lett. B 193, 207 (1987)

13. A. A. Tseytlin, Nucl. Phys. B 501, 41 (1997)

14. D. Brecher and M. J. Perry, Nucl. Phys. B 527, 121 (1998)

15. M. Born and L. Infeld, Proc. R. Soc. London A 143, 410 (1934)

16. M. Born and L. Infeld, Proc. R. Soc. London A 144, 425 (1934)

17. B. Hoffmann, Phys. Rev. 47, 877 (1935)

18. M. H. Dehghani and H. R. Rastegar-Sedehi, Phys. Rev. D 74, 124018 (2006)

19. D. L. Wiltshire, Phys. Rev. D 38, 2445 (1988)

20. M. Aiello, R. Ferraro and G. Giribet, Phys. Rev. D 70, 104014 (2004)

21. M. H. Dehghani and S. H. Hendi, Int. J. Mod. Phys. D 16 , 1829 (2007)

22. M. H. Dehghani, S. H. Hendi, A. Sheykhi and H. R. Rastegar-Sedehi, JCAP, 02, 020 (2007)

23. M. H. Dehghani, N. Alinejadi and S. H. Hendi, Phys. Rev. D 77, 104025 (2008)

24. S. H. Hendi, J. Math. Phys. 49, 082501 (2008)

25. M. Hassaine and C. Martinez, Phys. Rev. D 75, 027502 (2007)

26. S. H. Hendi and H. R. Rastegar-Sedehi, Gen. Relativ. Gravit. 41, 1355 (2009)

27. S. H. Hendi, Phys. Lett. B 677, 123 (2009)
28. M. Hassaine and C. Martinez, Class. Quantum Gravit. 25, 195023 (2008)

29. H. Maeda, M. Hassaine and C. Martinez, Phys. Rev. D 79, 044012 (2009)

30. S. H. Hendi and B. Eslam Panah, Phys. Lett. B 684, 77 (2010)

31. S. H. Hendi, Phys. Lett. B 690, 220 (2010)

32. S. H. Hendi, Prog. Theor. Phys. 124, 493 (2010)

33. S. H. Hendi, Eur. Phys. J. C 69, 281 (2010)

34. S. H. Hendi, Phys. Rev. D 82, 064040 (2010)

35. E. Ayon-Beato and A. Garcia, Phys. Rev. Lett. 80, 5056 (1998)

36. H. P. de Oliveira, Class. Quantum Gravit. 11, 1469 (1994)

37. H. H. Soleng, Phys. Rev. D 52, 6178 (1995)

38. M. H. Dehghani and M. Shamirzaie, Phys. Rev. D 72, 124015 (2005)

39. M. H. Dehghani and R. B. Mann, Phys. Rev. D 73, 104003 (2006)

40. J. Maldacena, Adv. Theor. Math. Phys. 2, 231 (1998)

41. M. H. Dehghani, N. Bostani, and A. Sheykhi, Phys. Rev. D 73, 104013 (2006)

42. J. D. Brown and J. W. York, Phys. Rev. D 47, 1407 (1993)

43. J. D. Beckenstein, Phys. Rev. D 7, 2333 (1973)

44. S. W. Hawking, Nature 248, 30 (1974)

45. G. W. Gibbons and S. W. Hawking, Phys. Rev. D 15, 2738 (1977)

46. C. J. Hunter, Phys. Rev. D 59, 024009 (1999)

47. S. W. Hawking, C. J. Hunter and D. N. Page, Phys. Rev. D 59, 044033 (1999)

48. R. B. Mann, Phys. Rev. D 60, 104047 (1999)

49. R. B. Mann, Phys. Rev. D 61, 084013 (2000)

50. D. Kastor, S. Ray and J. Traschen, Class. Quantum Gravit. 27, 235014 (2010)

51. J. Oliva and S. Ray, Class. Quantum Gravit. 27, 225002 (2010)

52. R. C. Myers and B. Robinson, J. High Energy Phys. 08, $067(2010)$

53. M. H. Dehghani, etal., arXiv:1109.4708 
